Musées, Patrimoine et Culture scientifiques et techniques

$153 \mid 2014$

mai-juin 2014

\title{
Le récolement au muséum de Rouen
}

\section{Sébastien Minchin}

URL : http://journals.openedition.org/ocim/1390

DOI : $10.4000 /$ ocim. 1390

ISSN : 2108-646X

Éditeur

OCIM

Édition imprimée

Date de publication : 25 juin 2014

ISSN : 0994-1908

\section{Référence électronique}

Sébastien Minchin, «Le récolement au muséum de Rouen », La Lettre de I'OCIM [En ligne], 153 | 2014, mis en ligne le 25 juin 2016, consulté le 21 avril 2019. URL : http://journals.openedition.org/ocim/1390 ; DOI : 10.4000/ocim. 1390 


\section{Le récolement au muséum de Rouen}

\section{Sébastien Minchin}

Directeur du muséum d'Histoire naturelle de Rouen

Créé en 1828, le muséum de Rouen possède des collections qui n'ont jamais été inventoriées de façon exhaustive. Comme pour nombre de muséums, l'inventaire des collections et les connaissances s'y rapportant se résumaient à une affaire de spécialistes, transmise oralement de génération en génération, dans le meilleur des cas. L'établissement rouennais est pour cela un cas étonnant puisqu'il n'a connu que 3 directeurs en 137 ans (1828-1965).

Rouvert en 2007, après 10 années de fermeture pour raisons de sécurité, le muséum de Rouen axe son projet scientifique et culturel autour du principe du muséum durable et responsable : chaque action engagée s'inscrit dans le temps et le développement durable, rendant le musée acteur des sociétés. Ainsi, bien qu'étant issues majoritairement du XIX $\mathrm{X}^{\mathrm{e}}$ siècle, les collections deviennent de formidables vecteurs de compréhension et de questionnement sur l'évolution du muséum tout au long de son histoire.

\section{De l'inventaire au muséum durable et responsable}

Le muséum durable et responsable, c'est tout d'abord s'interroger sur le choix des objets à inventorier et entrant en collection. C'est se fixer une ligne de conduite claire quant au devenir des objets, notamment ceux définis par l'ICOM comme « objets sensibles ». Depuis 2007, le muséum de Rouen interroge ainsi la société sur la place et le statut des restes humains, tant du point de vue patrimonial qu'éthique, mais également sur la prise en compte des peuples autochtones et de leurs revendications, qu'elles soient de l'ordre de demandes de restitution ou simplement de la possibilité d'accès aux collections.

\section{L'inventaire comme témoin des conditions de conservation du passé}

Linventaire et le récolement nous renseignent sur l'état des collections et permettent de déceler les conditions inadaptées devant nous inciter à repenser nos pratiques de conservation : c'est passer du tout chimique et d'une conservation curative à une conservation préventive dynamique. À Rouen, le grand écart entre les très mauvaises conditions de conservation dues à l'absence de chauffage et la surprenante «bonne conservation » des collections nous a amenés à nous interro- ger sur les pratiques du passé quant à l'utilisation en grandes quantités de produits chimiques de traitement devant éviter les infestations.

\section{L'inventaire comme témoin des pratiques du passé}

Ce constat est à l'origine d'un programme de recherche novateur et ambitieux, dont les objectifs sont de détecter et d'analyser les produits chimiques utilisés tant lors des préparations des spécimens que lors des traitements successifs, et de déterminer si des résidus de ces produits sont encore présents sur les spécimens et dans l'environnement muséal. Les prémices de ce projet ont fort naturellement été testés lors de la restitution de la tête maorie à la Nouvelle-Zélande, afin d'éviter que celle-ci, potentiellement polluée, ne contamine le sol lors de sa future inhumation.

Depuis 2010, ce programme de recherche piloté par le muséum, est mené conjointement avec l'Institut National des Sciences Appliquées de Rouen (INSA) et son département de Maîtrise des Risques Industriels et Environnementaux (MRIE), le Centre de Recherche et de Restauration des Musées de France (C2RMF) et Air-Normand, organisme missionné par le ministère de l'Écologie pour analyser sur la région Haute-Normandie la pollution de l'air liée au trafic automobile et aux industries.

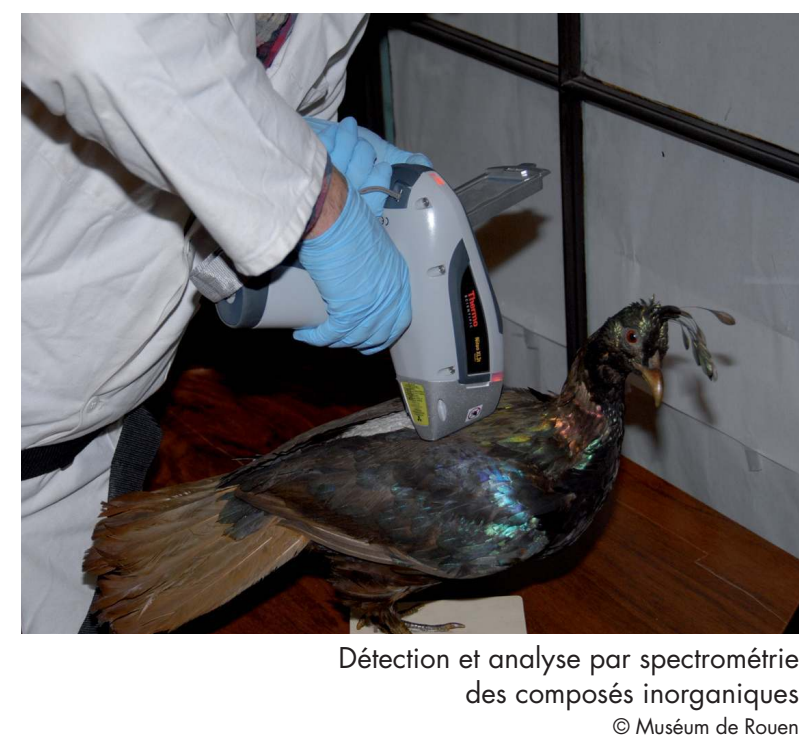




\section{Points de vue complémentaires}

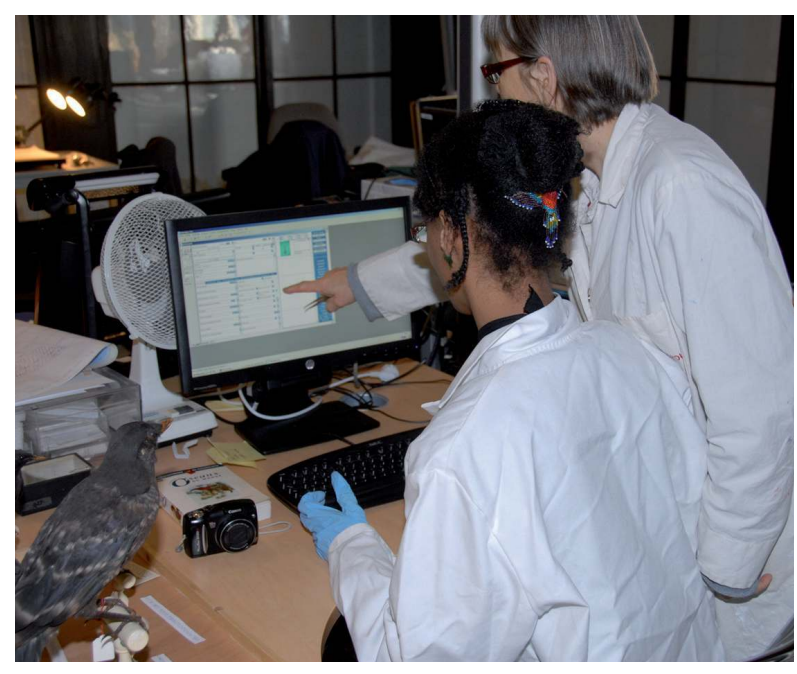

Saisie informatique des données d'inventaire (c) Muséum de Rouen

Chaque partenaire, selon son domaine de compétence et ses objectifs propres, trouve un intérêt indéniable à ce projet et $\mathrm{y}$ apporte son expertise et des moyens techniques importants : recherche et formation des élèves ingénieurs pour l'INSA, recherche en conservation et restauration des collections spécifiques pour le C2RMF, études et analyses de l'impact de ces polluants peu étudiés dans l'environnement pour AirNormand.

\section{Vers un inventaire durable et responsable}

Ce projet durable et responsable assure une meilleure connaissance des pratiques héritées du passé et améliore les conditions de travail et la prise en compte de la santé des agents.

En intégrant ces résultats aux inventaires et récolements, dans une sorte de "19e colonne virtuelle", nous construisons une traçabilité des polluants détectés et analysés, tant au niveau des spécimens que des vitrines et des bâtiments.

La transmission écrite de l'information et des actions entreprises, au travers de l'inventaire et du récolement, devient un investissement important pour le muséum, très éloigné de la logique d'antan principalement axée sur l'oralité. C'est toute la philosophie d'un muséum durable et responsable. Les sociétés évoluent, le musée aussi. 